\title{
Automated Attendance System Using Face Recognition Technique
}

\author{
Sumanta Chatterjee, Adit Jana, Adrija Ganguly, Aditi Ghosh
}

\begin{abstract}
The objective of this system is to present an automated system for human face recognition for an organization or institute to mark the attendance of their students or employees. This paper introduces face detection method using the Voila and Jones algorithm and recognition using correlation technique. The system will record the attendance of the students in class room environment. The above system is fully automated and easily deployable. User gets an authentication to upload the image containing file and also to view the attendance.
\end{abstract}

Index Terms - Automated, Face Detection, Face Recognition, Voila and Jones Algorithm, Correlation, Attendance.

\section{INTRODUCTION}

Face recognition is as old as computer vision, both because of the practical importance of the topic and theoretical interest from cognitive scientists. Despite the fact that other methods of identification (such as fingerprints, or iris scans) can be more accurate, face recognition has always remains a major focus of research because of its noninvasive nature and because it is people's primary method of person identification. Face recognition technology is gradually evolving to a universal biometric solution since it requires virtually zero effort from the user end while compared with other biometric options. Biometric face recognition is basically used in three main domains: time attendance systems and employee management; visitor management systems; and last but not the least authorization systems and access control systems. Traditionally, student's attendances are taken manually by using attendance sheet given by the faculty members in class, which is a time consuming event. Moreover, it is very difficult to verify one by one student in a large classroom environment with distributed branches whether the authenticated students are actually responding or not.

\section{PROPOSED SYSTEM ARCHITECTURE}

\section{A. Application layer}

There is the capturing phase in this the user captures the frames and using a web app that runs on almost all platforms upload the file to the server. Authentication is provided to the users. This web app is used to upload captured frames as well

Mr. Sumanta Chatterjee is presently working as an Assistant Professor of JIS College of Engineering, Kalyani, Nadia, West Bengal.

Adit Jana is a final year UG student of Computer Science and Engineering from JIS College of Engineering, Kalyani, Nadia, West Bengal. India

Adrija Ganguly is a final year UG student of Computer Science and Engineering from JIS College of Engineering, Kalyani, Nadia, West Bengal,India

Aditi Ghosh is a final year UG student of Computer Science and Engineering from JIS College of Engineering, Kalyani, Nadia, West Bengal. India as to view the attendance.

\section{B. System layer}

This is the layer where the processing is done that is the detection and recognition part at the server side. Viola and Jones algorithm is used to detect images from the frames. Initially an integral image is generated from the frame which simply assigns numbers to the pixels generated by summing up the values. Further to detect the objects from the frames the Haar-like feature is generated and as millions of features being generated Adaboost (boosting algorithm) is used to enhance the performance. The extracted features are passed through a trained classifier which detects the faces from the objects.

These detected faces are cropped and passed through the recognition module which by applying correlation to the cropped images and the images in the databases recognizes the faces.

\section{Database layer}

The Database layer is a centralized database system which consists of student database and their attendance. The student database is formed by initial feeding of the frames from which system detects faces crops them and stores it to the database and these stored images are hence forth used for the recognition part. The results of the face recognition module are compared with the images from the student database and after the successful comparison the attendance is updated to the database. The sheet is generated and uploaded to the web app.

\section{ALGORITHM}

\section{A. VOILA AND JONES ALGORITHM}

Viola and Jones algorithm is used for face detection. Where it is used in both creating database and face recognition process. Where in case creating database it takes input image through a web camera continuously. Captured image undergoes face detection. Detected face will be cropped and stored in database. Where in case of face recognition if there is any movement video surveillance will be used to detect the moving object. The captured image undergoes face detection and further processed later by face recognition. Cross-Correlation and Normalized-Correlation are used to extract the Coordinates of peak with the RIO and target images. The peak of the cross-correlation matrix occurs where the sub images are best correlated. Find the total offset between the images. The total offset or translation between images depends on the location of the peak in the cross correlation matrix, and on the size and position of the sub images. Check if the face is extracted from the target Image. Figure out where face exactly matches inside of target image. This algorithm mainly has following functionality. 


\section{Automated Attendance System Using Face Recognition Technique}

\section{CONCEPTUAL DIAGRAM}

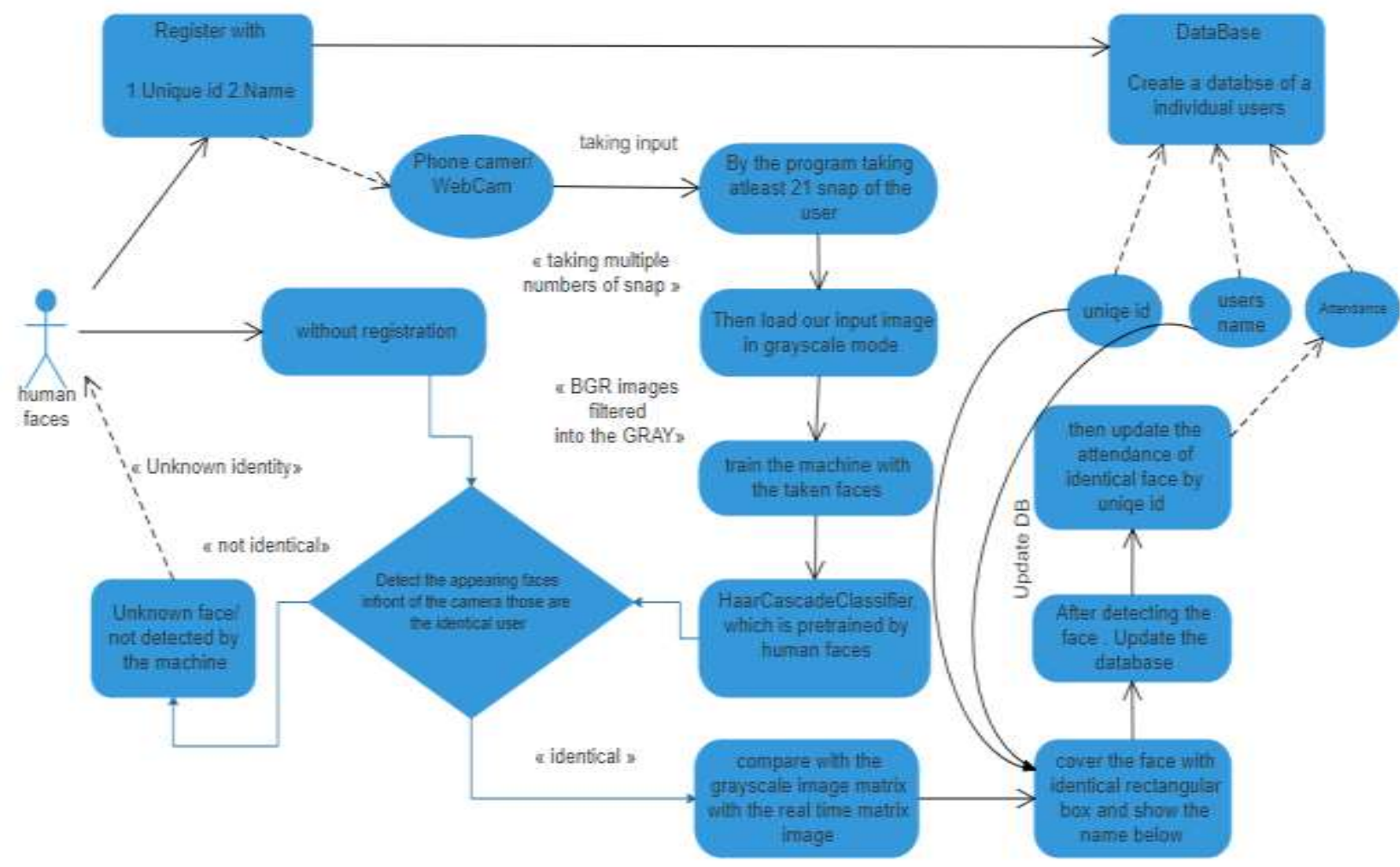

Fig A: Conceptual Diagram of the system
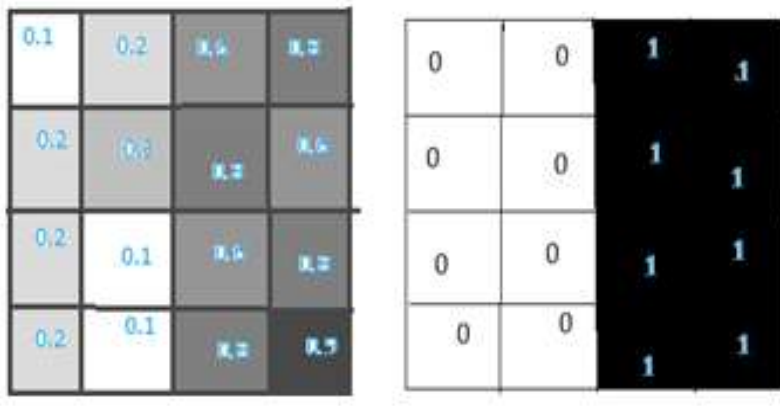

Fig B: color representation through matrix.

Ideal Haar-feature pixel intensities 0: white color, 1: black color. These are the real values to detect an image. Let's sum up the white pixel intensities. calculate the sum of the black pixel intensities.

$\Delta=$ dark - white

$\Delta=1 / n \sum_{\text {dark }}^{n} 1(x)-1 / n \sum_{\text {white }}^{n} 1(x)$

$\Delta$ for ideal Haar-feature is 1 .

$\Delta$ for the real image: $0.74-0.18=0.56$, The closer value to 1 , the more likely we have found a Haar-feature !!(of course we will never get 0 or 1 : there are thresholds).

\section{MECHANISM FOR FACE DETECTION}

\section{A. Face Detection}

Integral image or summed area table is a data structure and algorithm for quickly and efficiently generating the sum of values in a rectangular subset of a grid. In the image processing domain, it is also known as an integral image.
Haar-like features are digital image features used in object recognition. They owe their name to their intuitive similarity with haar wavelets and were used in the first real-time face detector. All human faces share some similar properties. These regularities may be matched using Haar Features.A few properties common to human faces: The eye region is darker than the upper-cheeks. The nose bridge region is brighter than the eyes.

Composition of properties forming matchable facial features: Location and size: eyes, mouth, bridge of nose Value: oriented gradients of pixel intensities the four features matched by this algorithm are then sought in the image of a face (shown at right).

Rectangle features:

Value $=\Sigma$ (pixels in black area) $-\Sigma$ (pixels in white area)Three types: two-, three-, four-rectangles, Viola \&amp; Jones used two-rectangle features, the difference in brightness between the white \&amp;black rectangles over a specific area Each feature is related to a special location in the sub-window Adaboost (adaptive boost) meta-algorithm formulated by yoav freund and robert schapire which is use to improve the performance of other algorithm. Viola and jones extracts the millions of features (pixels) for comparison so, we adaboost to enhance the overall performance and calculation speed of the algorithm.

Cascade classifier is a particular case of ensemble learning based on the concatenation of several classifiers, using all information collected from the output from a given classifier as additional information for the next classifier in the cascade. 
Unlike voting or stacking ensembles, which are multi-expert systems, cascading is a multistage one.

\section{B. Face Recognition}

Initially the ROI is extracted from the source face image, ROI is the sub image and is smaller than the original image. Normalized Cross-Correlation is performed on ROI and target image to find the peak coordinates. The total offset or translation is carried out based on the position of the peak in the cross correlation matrix. Check for the successful extraction of face from target image and figure out where the face exactly matches inside of target image.

\section{RECTANGULAR HAAR-LIKE FEATURES}

A simple rectangular Haar-like feature can be defined as the difference of the sum of pixels of areas inside the rectangle, which can be at any position and scale within the original image. This modified feature set is called 2-rectangle feature. Viola and Jones also defined 3-rectangle features and 4-rectangle features. The values indicate certain characteristics of a particular area of the image. Each feature type can indicate the existence (or absence) of certain characteristics in the image, such as edges or changes in texture. For example, a 2-rectangle feature can indicate where the border lies between a dark region and a light region edge feature can detect edges quite effectively; line feature can detect lines quite effectively.
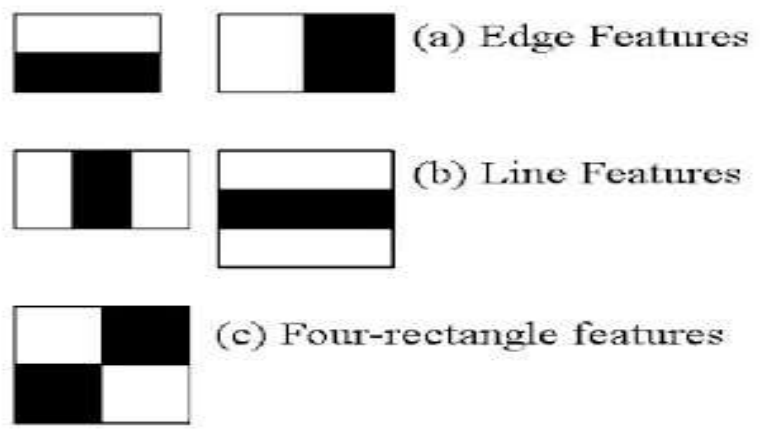

(c) Four-rectangle features

Fig C: rectangular haar-like features

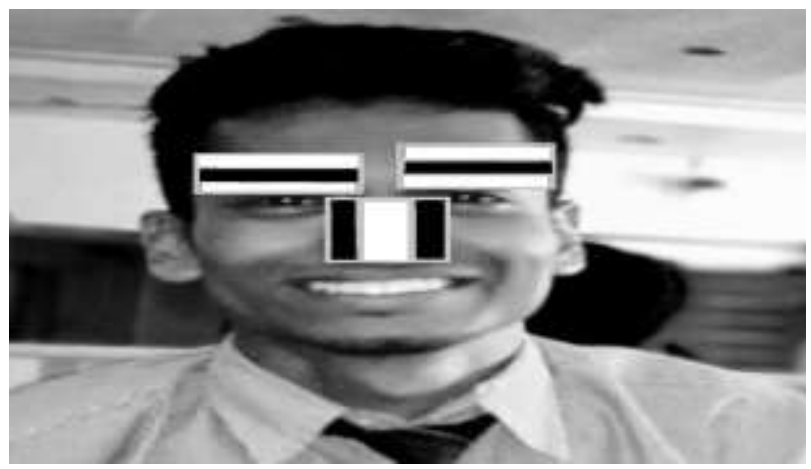

Fig D: Detection of the position of nose, eyebrows and mouth.

\section{STEP BY STEP PROCESS}

Exact process step by step:

- At first executable file take the faces.

- Then we train the machine.

- Then the image convert to the gray image.
- The numpy convert a huge matrix form of the image by the Voila-Jones Algorithm.

- Then The machine checks the exact matrix format of the already taken photos and match

- If the faces match then totally detect the faces

\section{A. Cascade Training:}

After the initial algorithm, it was understood that training the cascade as a whole can be optimized, to achieve a desired true detection rate with minimal complexity. Examples of such algorithms are RCBoost, ECBoost or RCECBoost. This can be used for rapid object detection of more specific targets, including non-human objects with Haar-like features. The process requires two sets of samples: negative and positive, where the negative samples correspond to arbitrary non-object images. The time constraint in training a cascade classifier can be circumvented using cloud-computing methods.

\section{B. Cascade Detection:}

After dealing with training We have to take the face and also detect them. Cascade classifiers are available in OpenCV, with pre-trained cascades for frontal faces and upper body. When we add eye detect classifier(haarcascade_eye.xml) then it detects the eye also.

\section{Tool Kits:}

\section{Matplolib:}

Matplotlib is a python 2D plotting library which produces publication quality figures in a variety of hard copy formats and interactive environments across platforms. Matplotlib can be used in Python scripts.

Numpy:

Numpy is a library for the Python Programming language, adding support for large multi-dimensional matrices and array, along with a large collection of high level mathematical function to operate on these arrays. It's a numerical python module.

OpenCV :

For open cv now the coding for the facial recognition is easier than ever in open cv there are three easy steps for the coding of facial recognition. That is similar to the how us brain used to recognize the face.

- Data Gathering: gather the facial data by useful algorithms.

- Train the recognizer: feed the facial data and unique id so that the recognizer can detect.

- Recognition: take the new faces and test it how recognizer can recognize the face or not.

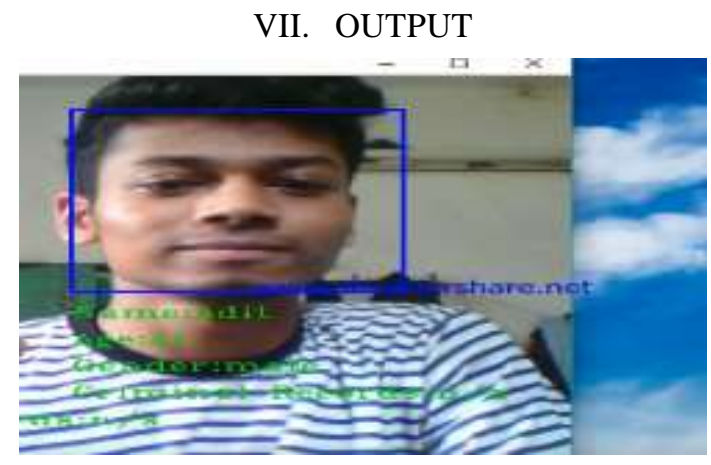

Fig E: image after recognition with exact id, name and roll no, the blue rectangle indicates the area of detected face. 


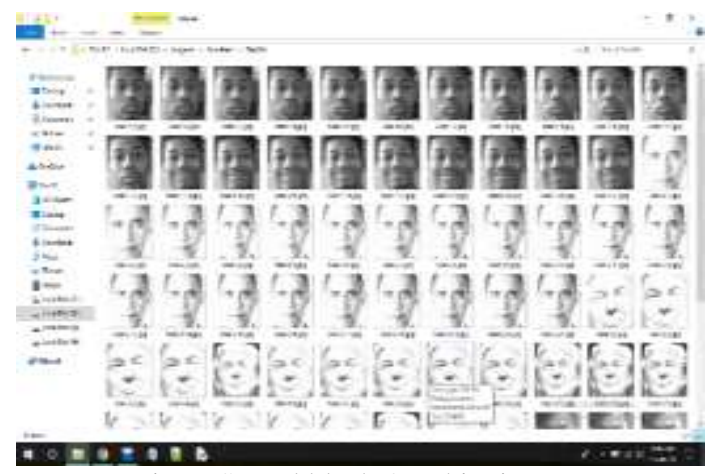

Fig F: Stored black \& White images

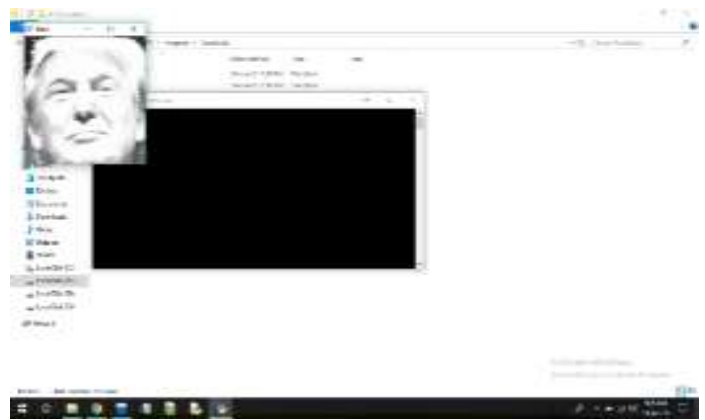

Fig G: Train images in the Datasets

\section{CONCLUSION}

In order to obtain the attendance of individual and to record their time of entry and exit, the authors proposed the attendance management system based on face recognition technology in the institutions/organizations. The system takes attendance of each student by continuous observation at the entry and exit points. The result of our preliminary experiment shows improved performance in the estimation of the attendance compared to the traditional black and white attendance systems. Current work is focused on the face detection algorithms from images or video frames.

\section{REFERENCES}

[1] A. J. Goldstein, L. D. Harmon, and A. B. Lesk, "Identification of Human Faces," in Proc. IEEE Conference on Computer Vision and Pattern Recognition, vol. 59, pp 748 - 760, May 1971

[2] M. A. Fischler and R. A. Elschlager, "The Representation and Matching of Pictorial Structures," IEEE Transaction on Computer,vol. C-22, pp. 67-92, 1973.

[3] Y. Cui, J. S. Jin, S. Luo, M. Park, and S. S. L. Au, “Automated Pattern Recognition and Defect Inspection System," in proc. 5 th International Conference on Computer Vision and Graphical Image, vol. 59, pp. 768 - 773, May 1992.

[4] M. H. Yang, N. Ahuja, and D. Kriegmao, "Face recognition using kernel eigenfaces," IEEE International Conference on Image Processing, vol. 1, pp. 10-13, Sept. 2000.

[5] Y.-W. Kao, H.-Z. Gu, and S.-M. Yuan "Personal based authentication by face recognition," in proc. Fourth International Conference on Networked Computing and Advanced Information Management, pp 81-85, 2008.

[6] P. Sinha, B. Balas, Y. Ostrovsky, and R. Russell, "Face Recognition by Humans: Nineteen Results All Computer Vision Researchers Should Know About," in Proceedings of the IEEE, vol. 94, Issue $11,2006$.

[7] Paul Viola, Michael Jones, 'Rapid Object Detection using a Boosted Cascade of Simple Features', Accepted Conference on Computer Vision and Pattern Recognition, 2001

[8]

FacedetectionWikipedialhttps://en.wikipedia.org/wiki/Face_detection

[9] Face detection - facedetection.com.
[10] Inseong Kim, Joon Hyung Shim and Jinkyu Yang (2016)Face Detection, Stanford University, International Journal of Engineering Research and Applications, Vol. 6, Issue 1, pp145-150.

[11] tutroals.readthedocs.io/en/latest/py_tutorials/py_objdete ct/py_face_detection/py_face_detection.html?highlight=opencv $\% 20 \mathrm{f}$ ace\#haar-cascade-detection-in-opencv

[12] R.O. Duda, P.E. Hart, Pattern Classification and Scene Analysis., New York::, 1973.

[13] W.E.L. Grimson, T. Lozano-Perez, \&quot;Model-Based Recognition and Localization From Sparse Range Data\&quot;,Techniques for 3-D Machine Perception., 1985.

[14] M. Turk, A. Pentland, \&quot;Eigenfaces for Recognition\&quot;,J. Cognitive Neuroscience, vol. 3, no. 1, pp. 71-86, 1991.

[15] A.Yuille,P.Hallinan, D. Cohen, \&quot;Feature ExtractionFromFacesUsingDeformableTemplates\&quot; Int\&\#39;1 J. Computer Vision, vol. 8, no. 2, pp. 99-111, 1992.

[16] K. Sung,Learning and Example Selection forObject and Pattern Detection, 1995

[17] D. Rumelhart, J. McClelland, Parallel Distributed Processing, Cambridge, Mass.::, vol. 1, 1986.

[18] T. Poggio, T. Vetter, \&quot;Recognition andStructure From One (2D) Model View:Observations on Prototypes Object Classesand Symmetries\&quot;, 1992.

\section{AUTHORS PROFILE:}

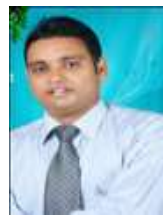

Mr. Sumanta Chatterjee is presently working as an Assistant Professor of JIS College of Engineering, Kalyani, Nadia, West Bengal. He has worked 2 years in the Industry and 6 years in the Academic Sector. He completed his M.Tech degree in Computer Science and Engineering and B.Tech degree in Information Technology from West Bengal University of Technology. He is recently working on the emerging research field "E-Commerce ". He has made significant contribution on the research field "Ecommerce".. He is a member of International Association of Computer Science and Information Technology (IACSIT) and also a member of International Association for Engineers (IAENG).

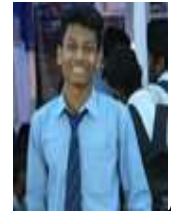

Adit Jana is a final year UG student of Computer Science and Engineering from JIS College of Engineering, Kalyani, Nadia, West Bengal. India

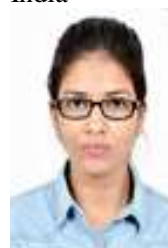

Adrija Ganguly is a final year UG student of Computer Science and Engineering from JIS College of Engineering, Kalyani, Nadia, West Bengal,India

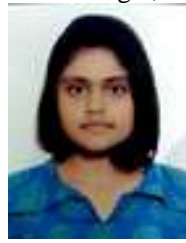
and Engineering from JIS College of Engineering, Kalyani, Nadia, West Bengal. India 